\title{
High-Resolution Single-Grain Diffraction of Polycrystalline Materials
}

\author{
Lienert, Ulrich; Ribárik, Gábor; Ungar, Tamas; Wejdemann, Christian; Pantleon, Wolfgang
}

Published in:

Synchrotron Radiation News

Link to article, DOI:

$10.1080 / 08940886.2017 .1316130$

Publication date:

2017

Document Version

Peer reviewed version

Link back to DTU Orbit

Citation (APA):

Lienert, U., Ribárik, G., Ungar, T., Wejdemann, C., \& Pantleon, W. (2017). High-Resolution Single-Grain Diffraction of Polycrystalline Materials. Synchrotron Radiation News, 30(3), 35-40.

https://doi.org/10.1080/08940886.2017.1316130

\section{General rights}

Copyright and moral rights for the publications made accessible in the public portal are retained by the authors and/or other copyright owners and it is a condition of accessing publications that users recognise and abide by the legal requirements associated with these rights.

- Users may download and print one copy of any publication from the public portal for the purpose of private study or research.

- You may not further distribute the material or use it for any profit-making activity or commercial gain

- You may freely distribute the URL identifying the publication in the public portal

If you believe that this document breaches copyright please contact us providing details, and we will remove access to the work immediately and investigate your claim. 


\title{
High Resolution Single Grain Diffraction of Polycrystalline Materials
}

\author{
U. Lienert ${ }^{1}$, G Ribárik ${ }^{2}$, T. Ungár ${ }^{3}$, C. Wejdemann ${ }^{4,5}$ and W. Pantleon ${ }^{5}$ \\ ${ }^{1}$ Photon Science Division, Deutsches Elektronen-Synchrotron, 22607Hamburg, Germany \\ ${ }^{2}$ Department of Materials Physics, Eötvös University, H-1117 Budapest, Hungary \\ ${ }^{3}$ Materials Performance Centre, School of Materials, The University of Manchester, Manchester M13 \\ $9 P L, U K$ \\ ${ }^{4}$ Roskilde Katedralskole, Holbakvej 59, 4000 Roskilde, Denmark \\ ${ }^{5}$ Department of Mechanical Engineering, Technical University of Denmark, 2800 Kongens Lyngby, \\ Denmark
}

Polycrystalline bulk materials are ubiquitous in everyday life including biological, geological, and engineered structural and functional materials. Their fundamental units are individual grains which are characterized by their microstructure, i.e. the arrangement of lattice defects. The microstructure usually influences the materials properties critically.

It has been demonstrated that by using high-energy synchrotron radiation, diffraction peaks off individual grains can be recorded in-situ during processing [1, 2]. Important information such as the orientation, average strain, and size of individual grains can be obtained even if the peak shapes are commonly not analyzed. However, it is also well known that the shape of diffraction peaks, if observed with sufficient resolution, contains significant information about the microstructure. While the intensity distribution in reciprocal space of a perfect lattice consists of delta functions located at the reciprocal lattice points, defects induce characteristic peak broadening. For exploiting the wealth of microstructural information contained in broadened diffraction peaks, the intensity distribution has to be characterized in all three-dimensions of reciprocal space. Distinguished directions are the radial direction, parallel to the reciprocal lattice vector $\mathbf{g}$ and quantified by differences in the scattering angle $2 \theta$, and the azimuthal directions, perpendicular to the reciprocal lattice vector and quantified by the angles $\eta$ and $\omega$ (cf. Figure 1). Conventional radial profile (line shape) analysis techniques average over many grains with possibly significantly different microstructure. Under conditions of single grain diffraction these limitations are overcome and the intensity distributions along all three directions of reciprocal space are accessible.

Large reciprocal space coverage is desirable since the anisotropy of lattice defect arrangements can be characterized if a sufficient number of projections (reciprocal lattice vectors) are measured. High resolution is desirable as individual subgrains become discernable. However, since the number of independent pixels of available detectors is limited, two distinct experimental setups have been developed and the respective methodologies and case studies are described below.

For a moderately high resolution, $\Delta \mathrm{g} / \mathrm{g} \approx 10^{-3}$ ( $\mathrm{g}=2 \sin \theta / \lambda$ indicating the length of the diffraction vector), a large reciprocal space coverage can be realized (all reflections from lattice planes with interplanar spacings above about 1 Á, see section on large reciprocal space coverage with high angular resolution), through emulating a large detector by translating a single detector to a few adjacent grid positions. The line profile data presented here have been recorded at 1-ID at APS with a sample-to-detector distance of $2.5 \mathrm{~m}$.

For an even higher resolution, $\Delta \mathrm{g} / \mathrm{g} \approx 10^{-4}$ in all three dimensions, a dedicated set-up, limited to a single diffraction peak (see section on high resolution reciprocal space mapping), has been realized at 
beam line 1-ID at APS [3] and recently recreated at beam line P07 at PETRA III [4]. A highly monochromatic and partially focused beam of low divergence is utilized, and a detector with small pixel size is placed about $4 \mathrm{~m}$ behind the sample.

\section{Large reciprocal space coverage with high angular resolution}

The substructure of polycrystalline bulk materials is usually heterogeneous on different length scales: Within individual grains it is heterogeneous on the subgrain scale $[6,7]$ and within the bulk polycrystal it is heterogeneous on the grain scale [8]. In hexagonal close packed ( $h c p)$ materials an additional heterogeneity prevails because of the many different slip modes and Burgers vector types [9]. On all scales all microstructural properties, e.g. dislocation densities, slip modes, Burgers vector types and populations, planar defect types and densities, subgrain size or subgrain orientation distribution, can vary and can be heterogeneous. The different types of heterogeneities have very different implications on the different behavior and performance of materials $[10,11,12]$. Conventional X-ray diffraction experiments on bulk polycrystalline samples provide average information about the volume illuminated by the beam. In the following the principles of a novel synchrotron procedure are described which characterizes the substructure of a large number of individual grains in bulk polycrystalline aggregates. In this procedure, averaging is restricted to the individual grains despite that the illuminated volume in the sample comprises of several hundred grains.

The 3D grain mapping procedure $[1,13]$ was extended to determine the microstructure of individual grains in polycrystalline aggregates $[14,15,16,17]$. Dislocation densities, slip modes, Burgers vector populations, subgrain size and planar defects were determined in large number of individual grains in bulk polycrystalline specimens of tensile deformed $\mathrm{CoTi}$ and $\mathrm{CoZr}$ alloys [17], in an $\mathrm{MgGeO}_{3}$ postperovskite [15] and in stishovite synthesized in-situ in diamond anvil cells at the synchrotron beamline [16]. The specimens are either free standing thin needle-shape rods in atmospheric ambient surroundings or kept under high pressure in diamond anvil cells with large openings. The free standing specimens are rotated at least $180^{\circ}$ around a single axis. The specimens at high pressure are turned around the rotation axis by angles permitted by the openings of the anvil cell. Diffraction images are recorded with two dimensional detectors at a closer and a farther distance. The close detector images are used to determine the orientation matrices of the grains in the $\mathrm{X}$-rays illuminated volume. The angular resolution achieved for the images obtained by the far detector is good enough to carry out line profile analysis.

Diffraction line profile analysis proves to be a powerful tool for determining the sub-structure of crystalline materials $[18,19,14,20]$. The convolutional multiple whole profile (CMWP) procedure [14] is based on physically modeled profile functions for the different microstructural elements. Microstrain is assumed to be produced by the presence of dislocations and the corresponding strain profiles are built up using the Wilkens model [21]. The Fourier transform of the strain profiles, $A_{h k l}^{D}(L)$, are:

$A_{h k l}^{D}(L)=\exp \left(-2 \pi^{2} L^{2} g^{2}\left\langle\varepsilon_{g, L}^{2}\right\rangle\right)$, 
where $L$ is the Fourier variable and $\left\langle\varepsilon_{g, L}^{2}\right\rangle$ is the means square strain. For dislocations $\left\langle\varepsilon_{g, L}^{2}\right\rangle$ is [21]:

$$
\left\langle\varepsilon_{g, L}^{2}\right\rangle=\frac{\rho C_{h k l} \mathrm{~b}^{2}}{4 \pi} f\left(\frac{L}{R_{e}}\right)
$$

where $\rho, C_{h k l}$ and $\mathrm{b}$ are the density, the $h k l$ dependent contrast factor and the Burgers vector of dislocations, respectively. The $f\left(\frac{L}{R_{e}}\right)$ function is logarithmic at small $L$ values and hyperbolic at large $L$ values. In a conventional powder diffraction pattern averaging over the permutations of the Miller indices $h k l$ obscures the orientation dependence of dislocation contrast. Such experiments provide dislocation densities or Burgers vector population averaged over polycrystalline aggregates [22, 23]. One of the aims of single grain diffraction experiments is to obtain dislocation densities and Burgers vector populations on the level of individual grains. Elastic strains along the diffraction vector essentially broaden the radial intensity distribution, whereas lattice rotations or shear strains blur the intensity distribution in the perpendicular, i.e. the azimuthal directions. The first is usually called line broadening, whereas the latter is known as rocking curve broadening [21]. The dislocation density and Burgers vector population is provided by line broadening [21, 24]. In a single crystal diffraction experiment the three dimensional intensity distribution, $I_{h k l}(2 \theta, \omega, \eta)$, is integrated over the azimuthal intensity distributions, i.e. $\omega$ and $\eta[14]$ :

$$
I_{h k l}(2 \theta)=\int I_{h k l}(2 \theta, \omega, \eta) d \omega d \eta
$$

The technical details about determining the orientation matrix and integration of the intensity distributions over $\omega$ and $\eta$ can be found in references $[14,17]$.

Analysis of the strain anisotropy in powder diffraction proved to be a powerful method to determine average Burgers vector populations in polycrystalline materials $[25,26]$. The single grain procedure extends this method to single crystals. It is based on matching measured contrast values, $C_{h k l}^{\text {meas }}$, with theoretical contrasts, $C_{h k l}^{\text {theor }}$, where the indices $h k l$ are orientation dependent, signed values.

We can assume that the total dislocation density, $\rho$, consists of the sum of the fractional dislocation densities, $\rho_{\text {fract }}\left(\boldsymbol{b}_{n}, \boldsymbol{I}_{n}\right)$, where their broadening effect is scaled with the corresponding Burgers vectors, $\boldsymbol{b}_{n}$, and contrast factors, $C_{\text {theor }}\left(\boldsymbol{g}, \boldsymbol{b}_{n}, \boldsymbol{l}_{n}, \mathrm{c}_{\mathrm{i}, \mathrm{j}}\right)$. The subscript in $C$ refers to theoretically calculated contrast factors [27], $I_{n}$ are the line vectors of dislocations and $c_{i, j}$ are the elastic constants of the material. The strain profiles in each measured diffraction peak provide average, measured contrasts, $C_{C M W P}\left(\boldsymbol{g}_{h k}\right)$, where the subscript refers to values provided by the CMWP procedure. The measured and theoretically calculated contrasts are matched by minimizing the difference between the measured and calculated mean square strain values [17]:

$$
\left[\sum_{b_{n}, l_{n}} \rho_{\text {fract }}\left(\boldsymbol{b}_{\boldsymbol{n}}, \boldsymbol{l}_{\boldsymbol{n}}\right) b_{n}^{2} C_{\text {theor }}\left(\boldsymbol{g}, \boldsymbol{b}_{\boldsymbol{n}}, \boldsymbol{l}_{\boldsymbol{n}}, c_{i, j}\right)-\rho_{C M W P} b_{C M W P}^{2} C_{C M W P}\left(\boldsymbol{g}_{\boldsymbol{h k} \boldsymbol{k}}\right)\right]=\min ,
$$

where $b_{C M W P}$ is a formal input value for running the CMWP software [14]. Once the difference in eq. (4) has been minimized the following relation is obtained:

$$
C_{C M W P}\left(\boldsymbol{g}_{h k l}\right) \cong \sum_{b, l} \kappa_{b, l}^{2} C_{\text {theor }}\left(\boldsymbol{g}, \boldsymbol{b}_{h k l}, \boldsymbol{l}_{h k l}, c_{i, j}\right),
$$


where $\kappa_{b_{n}, l_{n}}^{2}=\frac{\rho_{\text {part }}\left(\boldsymbol{b}_{n}, \boldsymbol{l}_{\boldsymbol{n}}\right) b^{2}}{\rho_{C M W P} b_{C M W P}^{2}}$ is the scaling factor between the measured and theoretically calculated contrast factors. The fractional dislocation densities in the different slip systems are obtained as:

$\rho_{\text {fract }}\left(\boldsymbol{b}_{n}, \boldsymbol{l}_{n}\right)=\frac{\kappa_{,, l}^{2}}{b^{2}} \rho_{C M W P} b_{C M W P}^{2}$.

The procedure is illustrated by an example:

Single crystals of CoTi and CoZr intermetallics of the B2 type, $\mathrm{CsCl}$ structure, can only deform plastically if they are oriented for a particular slip-mode such that $\langle 001>\{110\}$ slip systems become activated $[28,29,30]$. When deformed in other orientations they respond with early brittle fracture $[31,28]$. The $<001>\{110\}$ slip-mode, often called soft-mode, provides only three independent slip systems. This would not be sufficient for the generalized criterion of the number of slip systems required to accommodate arbitrary strains in polycrystalline specimens [32, 33]. Despite the limited number of slip systems in the soft slip-mode, the polycrystalline specimens of these alloys exhibit significant ductility $[29,30]$. Lattice strain measurements by in-situ neutron diffraction experiments supported by polycrystal plasticity modeling have shown that during deformation of polycrystalline CoTi and CoZr the slip systems of the soft mode are accompanied by the activation of $\langle 110>\{110\}$ and/or $<111>\{111\}$ slip systems [29]. The latter are often called hard mode slip systems. Recent transmission and scanning electron microscope (TEM and SEM) and electron backscatter diffraction (EBSD) confirmed the presence of dislocations with $<110>$ and $<111>$-Burgers vectors [34]. However, only a small number of grains were analyzed and no quantitative dislocation density information was obtained. Analysis of the line broadening from synchrotron single grain experiments gave soft and hard slip-mode distributions along with partial and total dislocation densities in well above 100 grains in each specimen [17]. The partial and total dislocation densities in the CoTi alloy are shown in inverse pole figures in Figure 2. The results show that data which were formerly relegated to the transmission electron microscope are now available from this novel 3D synchrotron $x$-ray diffraction procedure. Since a very large number of grains was analyzed in the two B2 structure alloys, it can be concluded that plastic deformation in CoTi and CoZr is carried by the combined activation of softmode $(b=<100>)$ and hard-mode $(b=<110>$ and $\langle 111>)$ dislocations, in agreement with TEM observations of the same materials. The results provide an extensive experimental data base for polycrystal plasticity modeling.

\section{High resolution reciprocal space mapping}

High-resolution reciprocal space mapping (HRRSM) enables identification and monitoring of individual subgrains in the deformation microstructure of selected grains in polycrystalline materials $[3,35,37,36]$. Three-dimensional reciprocal space maps are achieved by acquiring a series of twodimensional intensity distributions while rocking through small, subsequent intervals $\Delta \omega$ of the rocking angle. A detector image obtained during rocking through a small rocking interval of $0.015^{\circ}$ from a single, selected grain in a tensile deformed polycrystalline sample of copper is shown in the insert of Figure 1. Owing to the high-angular resolution, the diffracted intensity does not manifest itself as broad, smooth distribution, rather distinct features emerge and several peaks of high intensity are revealed $[3,38]$. These high-intensity peaks are sharp in all three dimensions of reciprocal space, show neither broadening from microstrains, nor from their sizes and originate from 
almost dislocation-free volumes of about 1 to $2 \mu \mathrm{m}^{3}$ (from integrated intensity). As they share these characteristics (except the smaller size) with diffraction peaks from undeformed grains, they are considered to arise from individual subgrains of slightly different orientation [3, 38]. Polycrystalline material not developing ordered dislocation structures with dislocation walls separating dislocation free regions (e.g. non-cell forming metals as AIMg alloys) develop rather smooth intensity distributions without any high-intensity peaks [39].

The main advantage of HRRSM is that the technique enables not only a thorough characterization of an established deformation structure, but also to investigate the evolution of deformation structures in-situ during plastic deformation. For instance, the emergence of ordered dislocation structures during the course of deformation has been proven in uninterrupted tensile tests $[35,40]$. The technique has been exploited for uniaxial tension [3, 38], stress relaxation and unloading [42] where the evolution of subgrain structures is monitored in-situ during varying mechanical loads. The method can also be applied to pre-deformed specimens, which are mechanically loaded in-situ to follow the enforced changes in the deformation structure, for instance, when a pre-deformed specimen is re-loaded along a different direction [35, 5]. Due to the change in strain path, the originally developed structure becomes alien and has to be replaced by one conform to the new deformation conditions.

As an example, the particular case where the new tensile direction is orthogonal to the tensile axis of the pre-deformation is discussed [43, 40]. Dog-bone shaped specimens were cut from coarse-grained copper sheets pre-deformed by $5 \%$ in uniaxial tension such that the new tensile axis is perpendicular to the tensile direction of the pre-deformation. Grains with their crystallographic $\langle 100\rangle$ direction close to the new tensile axis were selected. The specimen was tensile deformed in-situ and reciprocal space maps of their 400 diffraction peak were acquired at a minimal elastic strain of $0.03 \%$ (i.e. essentially before any strain path change) and for eight strain increments to a total additional strain of $1 \%$ after the strain path change.

Figure 3 presents azimuthal projections from one grain before and after the strain path change. In each projection, individual subgrains can be clearly revealed due to their unique orientation. After $0.2 \%$ tensile deformation, only minor changes have occurred and a lot of the initially present highintensity peaks can still be identified. A completely new orientation distribution is observed after $1 \%$ tensile deformation, indicating that the initial subgrain structure has been replaced by one conform to the new deformation condition.

The corresponding radial profiles before and after the strain path change are shown in Figure 4(a). After the strain path change, the profile is shifted towards lower diffraction angles (due to the applied load causing elastic straining of the lattice), broadened (due to the increased dislocation density) and asymmetric with a tail at low diffraction angles. Such an asymmetry of the radial profile is characteristic for ordered deformation structures with dislocation walls of high dislocation density separating dislocation-depleted regions [44] and can be rationalized in terms of the composite model $[44,45]$ or its refined version [40]: compatible deformation of ordered deformation structures requires forward stresses in the dislocation walls and back stresses in the dislocation-depleted regions. These back stresses lead to elastic distortions with compressive elastic strains along the tensile direction (axial case) and tensile elastic strains in the perpendicular directions (side case)[37]. Compressive elastic strains cause shorter interplanar spacings. For diffraction vectors parallel to the tensile axis (axial case), the high-intensity peaks from individual subgrains hence arise at larger 
diffraction angles than the average position of the grain (cf. Fig. 4(a)). The peak maximum occurs at larger diffraction angles than the average; the tail at smaller diffraction angles is due to the dislocation walls under forward stresses.

Further support for this interpretation can be gained from the 80 largest subgrains (for the details of the procedure cf. [41]). The normal probability plot in Figure 4(b) reveals that their radial peak positions are Gaussian distributed and in average larger than the radial peak position of the grains after $1 \%$ tensile deformation, i.e. the individual subgrains all experience relative compressive elastic strains slightly different from each other. This is different for the 80 largest subgrains in the predeformed specimen, i.e. before the strain path change: their radial peak positions are similarly Gaussian distributed (with a lower variation), but occur at lower diffraction angles than the average of the grain indicating the presence of relative tensile elastic strains. These tensile elastic strains are a consequence of the pre-deformation and the circumstance that the initial tensile direction is perpendicular to the chosen diffraction vector (side case). This difference in elastic strain of the subgrains is reflected in the radial profile obtained before the strain path change in Figure 4(a) which is less broad and has its tail at higher diffraction angles. With increasing tensile strain after the strain path change, the asymmetry of the radial profile becomes reversed, due to the subgrains developing compressive elastic strains instead of the tensile elastic strains from the pre-deformation [37, 43]. This highlights, how reorganization of deformation structures in individual grains in polycrystalline specimens and details about individual subgrains can be revealed in-situ by high resolution reciprocal space mapping during a change in loading conditions, e.g. during strain path changes [37] or cyclic deformation [4].

\section{Conclusion and outlook}

Two techniques of single grain three-dimensional reciprocal space mapping by high-energy synchrotron radiation are described. They are distinguished by their resolution and reciprocal space coverage. Grains deep in the bulk can be investigated non-destructively in-situ during mechanical (or thermal) loading. At large reciprocal space coverage, a large number of reflections per grain can be recorded simultaneously. From the strain anisotropy, as evaluated from radial peak profiles, the dislocation densities on particular slip systems can be determined for a large number of grains. At very high resolution, only one reflection is investigated at a time, but diffraction off individual subgrains is discernable. The behavior of a significant number of subgrains can be characterized during mechanical loading revealing for instance the reorganization of deformation structure in individual grains during a change in loading conditions.

Dramatic improvements of the present temporal (and/or reciprocal space) resolution are anticipated from technological advances. First, flat panel detector arrays with ten thousand pixel diagonals are becoming a realistic option and efficient CdTe pixel detectors are becoming commercially available. Second, ongoing upgrades of high-energy synchrotron facilities by multi-bend achromat lattices promise a significant improvement of the beam emittance and brightness which would directly translate into intensity gains. Eventually, coherent diffraction might under favorable conditions enable three-dimensional spatial resolution within individual subgrains. However, the incoherent techniques presented here would not become obsolete due to the much faster data acquisition and optional averaging over entire grains if desirable. 


\section{Acknowledgements}

Use of the Advanced Photo Source was supported by the U.S. Department of Energy, Office of Science, Basic Energy Sciences under contract No. DE-AC02-06CH11357.

\section{References}

1. H.F. Poulsen, Three-Dimensional X-Ray Diffraction Microscopy, vol. 205 of Springer Tracts in Modern Physics, Springer (2004).

2. U. Lienert et al., JOM 63, 70-77 (2011).

3. B. Jakobsen et al., Science 312, 889-892 (2006).

4. A. Diederichs et al., submitted to Journal of Physics: Conference Series (2017).

5. C. Wejdemann, et al., IOP J. Phys. Conf. Ser. 240, 012610 (2010).

6. H. Mughrabi, Mater. Sci. Eng. 85, 15-31 (1987).

7. U.F. Kocks and H. Mecking, Progr. Mater. Sci. 48, 171-273 (2003).

8. X. Huang et al., Mater. Sci. Eng. A319-321, 237-241 (2001).

9. I.P. Jones and W.B.Hutchinson, Acta Metall. 29, 951-968 (1981).

10. L. S. Tóth et al., J. Eng. Mater. Technol. 124, 71-77 (2001).

11. J. Segurado et al., J. Plasticity 28, 124-140 (2012).

12. M. Knezevic et al., J. Mech. Phys. Sol. 61, 2034-2046 (2013).

13. E. M. Lauridsen et al., J. Appl. Christ. 34, 744-750 (2001).

14. G. Ribárik and T. Ungár, Mater. Sci. Eng. A 528, 112-121 (2010).

15. C. Nisr et al., Geophys. Res. 117, B03201 (2012).

16. C. Nisr et al., High Press. Res. 34(2), 158-166 (2014).

17. T. Ungár et al., Acta Mater. 71, 264-282 (2014).

18. T. Ungár et al., J. Appl. Cryst. 34, 298-310 (2001).

19. P. Scardi and M. Leoni, Acta Cryst. A58, 190-200 (2002).

20. L. Rebuffi et al., Sci. Reports, | DOI: 10.1038/srep20712 (2016).

21. M. Wilkens, Fundamental Aspects of Dislocation Theory, edited by J.A. Simmons, R. de Wit \& R. Bullough, Vol. II., Nat. Bur. Stand. (US) Spec. Publ. No. 317, 1195-1221 (1970).

22. T. Ungár and G. Tichy, Phys. Stat. Sol. A 147, 425-434 (1999).

23. J. Martinez-Garcia et al., Acta Cryst. A65, 109-119 (2009). 
24. Gy. Zilahi et al., J. Appl. Cryst. 48, 418-430 (2015).

25. Jr.R. Kuzel and P. Klimanek, J. Appl. Cryst. 22, 299-307 (1989).

26. T. Ungár and A. Borbély, Appl. Phys. Lett. 61, 3173-3175 (1996).

27. A. Borbély et al., J. Appl. Cryst. 36, 160-162 (2003).

28. D.B. Miracle, Acta Mater. 41, 649-84 (1993).

29. J.A. Wollmershauser et al., Metall. Mater. Trans. 41A, 1217-1219 (2010).

30. R.P. Mulay et al., Metall. Mater. Trans. 42A(1), 60-70 (2011).

31. T. Takasugi et al., Philos. Mag. A 61, 785-800 (1990).

32. R.Z. von Mises, Math. Mech. 8, 161-85 (1928).

33. G.I. Taylor, J. Inst. Met. 62, 307-324 (1938).

34. R.P. Mulay and S.R. Agnew, Acta Mater. 60, 1784-1794 (2012).

35. W. Pantleon et al., Mater. Sci. Engng. A 524, 55-63 (2009).

36. W. Pantleon et al., in: Strain and dislocation gradients from diffraction, R. Barabash and G. Ice (Eds.), Imperial College Press, London, 322-357 (2014).

37. C. Wejdemann et al., JOM 65, 35-43 (2013).

38. B. Jakobsen et al., Acta Mater. 55, 3421-3430 (2007).

39. B. Jakobsen et al., Scripta Mater. 56, 769-772 (2007).

40. W. Pantleon, et al., In: Proc. 31st Intern. Risoe Symp. On Materials Science (Ris $\varnothing$ DTU, Roskilde, Denmark), 79-100 (2010).

41. C. Wejdemann et al., In: Proc. 31st Intern. Risoe Symp. On Materials Science (Ris $\varnothing$ DTU, Roskilde, Denmark), 477-487 (2010).

42. B. Jakobsen et al., Phys. Stat. Sol. (a) 206, 21-30 (2009).

43. C. Wejdemann et al., Scripta Mater. 62, 794-797 (2010).

44. H. Mughrabi et al., Philos. Mag. A 53, 793-813 (1986).

45. H. Mughrabi, Acta Mater. 31, 1367-1379 (1983). 


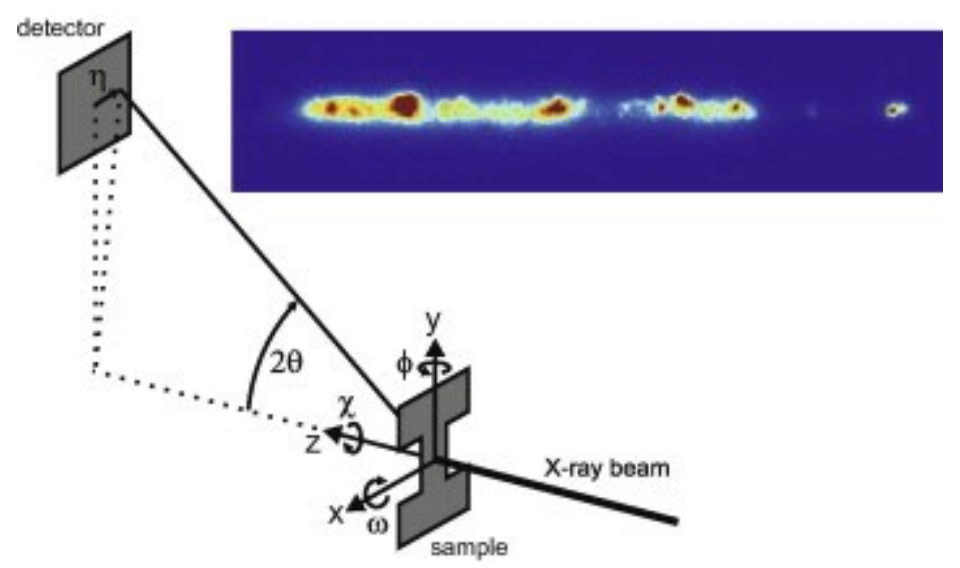

Figure 1: Principal sketch of the setup for three-dimensional high resolution reciprocal space mapping. The insert shows an intensity distribution along the radial direction $(2 \theta)$ and an azimuthal direction $(\eta)$. Mapping of the third dimension of reciprocal space is achieved by rocking $(\omega)$ in small intervals $(\Delta \omega)$. (from [5])
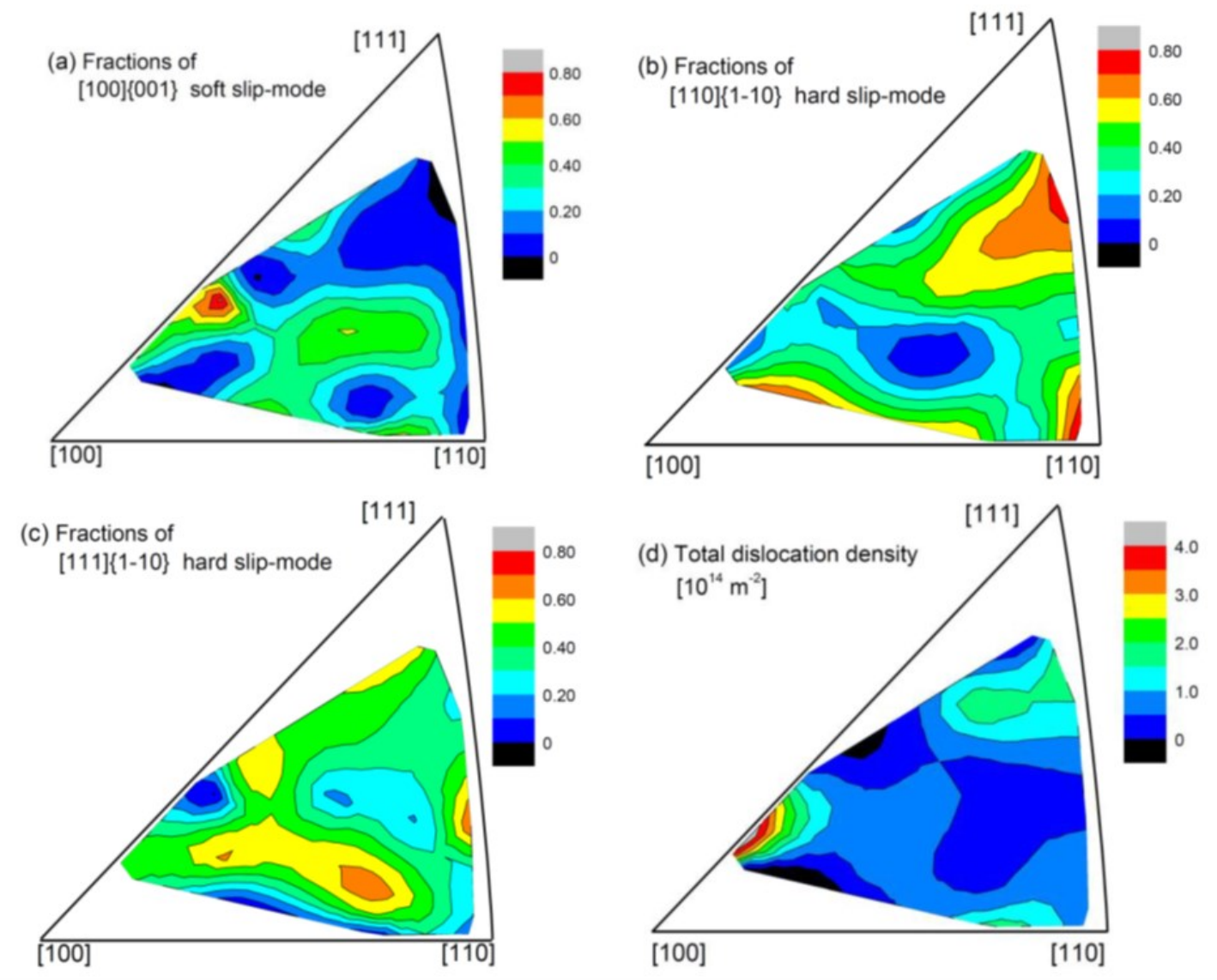

Figure 2: Slip mode fractions (a-c) and total dislocation densities (d) in 24 grains measured in one specimen section of the tensile deformed CoTi alloy [17]. The fractions of the [100]\{001\} type soft slip-mode (a), the [110]\{1-10\} type hard slip-mode (b) and the [111]\{1-10\} type hard slip-mode (c). (d) The total dislocation density. 
(a)
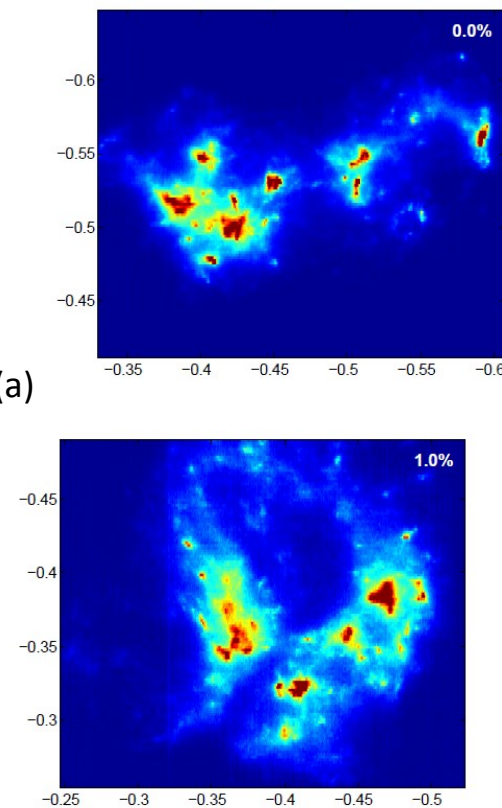

(b)

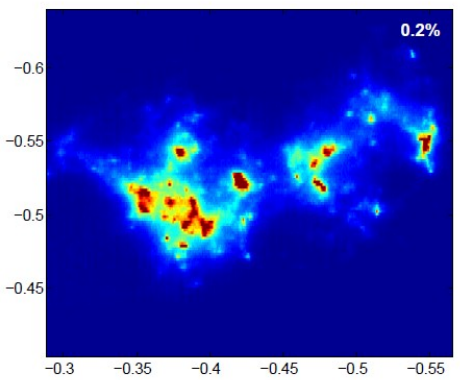

(c)

Figure 3: Azimuthal projections $(\eta, \omega)$ of the 400 diffraction peak from a single grain in a predeformed polycrystalline copper specimen (a) before (actually $0.03 \%$ ) and after a strain path change after further tensile deformation by (b) $0.2 \%$ and (c) $1 \%$.

(a)

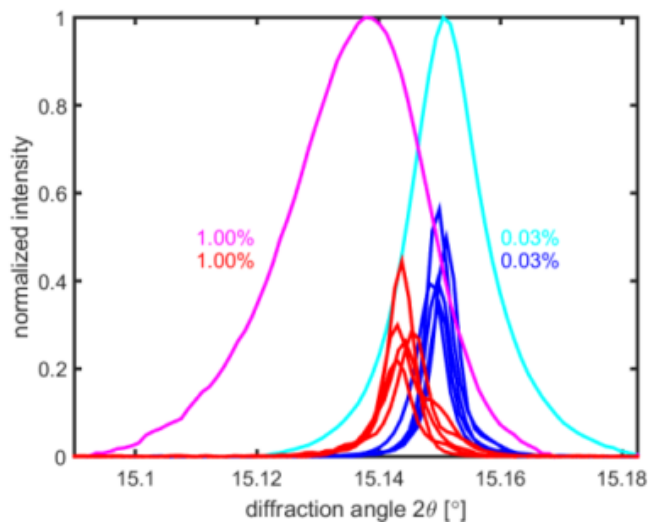

(b)

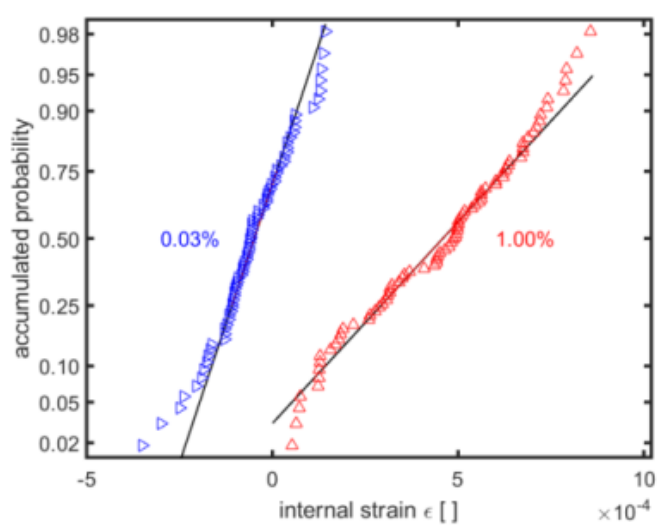

Figure 4: (a) Radial profiles (scaled to same maximum intensity) of the 400 diffraction peak from a single grain in a pre-deformed polycrystalline copper specimen before $(0.03 \%)$ and after a strain path change (1\%). Radial profiles (intensity scaled by 500 ) of high-intensity peaks corresponding to five individual subgrains are added in each case. (b) Radial peak position of the 80 most intense highintensity peak relative to the average position of the entire grain before $(0.03 \%)$ and after strain path change after tensile deformation. 